\title{
IV. Nanomaterials and nanostructures
}

A large part of the progress of human society is based on the development of materials. In the past metals have played a tremendous role in this respect. Progress in manufacturing techniques and the creation of new alloys have fostered advances in agriculture, transportation, warfare, cooking, etc. Various periods of rapid development in human history are identified by these advances: the age of copper, which occurred after the stone age, the bronze age, the age of iron. Today we are entering a new period corresponding to the age of nanomaterials. Creating new nanomaterials and understanding their properties will have far-reaching effects, Eiji Kobayashi, from Panasonic, says: "those who control materials control technology". This statement is certainly true for nanomaterials and their growing sociological and economic importance should not be underestimated.

\section{Classifying nanomaterials}

According to The International Organization for Standardization a "nanomaterial" is a material with any external dimensions in the nanoscale range or having internal structure or surface structure in the nanoscale range. This size range is typically considered to be from approximately $1 \mathrm{~nm}$ to $100 \mathrm{~nm}$. Nanomaterials are of particular interest because the unique properties, electrical, magnetic, optical etc., which can emerge on this size scale have wide application in a variety of fields.

It is possible to classify nanomaterials differently depending on which properties one focuses on, i. e., external or internal.

If attention is focused towards the external characteristics of a material, it is possible to classify it according to the external dimensions of the object. If only one dimension is smaller than $100 \mathrm{~nm}$, it is considered as a one-dimensional $(\mathrm{1D})$ nanomaterial. Ultra-thin films are an example of $1 \mathrm{D}$ nanomaterials. If 2 dimensions of the system are in the nanometer range, it is a two-dimensional nanomaterial (2D). Finally, if 3 of the dimensions of the system are smaller than $100 \mathrm{~nm}$ the material is a $3 \mathrm{D}$ nano-object. Nanoparticles or quantum dots belong to this category. Figure 26 summarizes some types of nanomaterials which can be produced and tailored to be utilized in applications such as those discussed in the remainder of this book. 


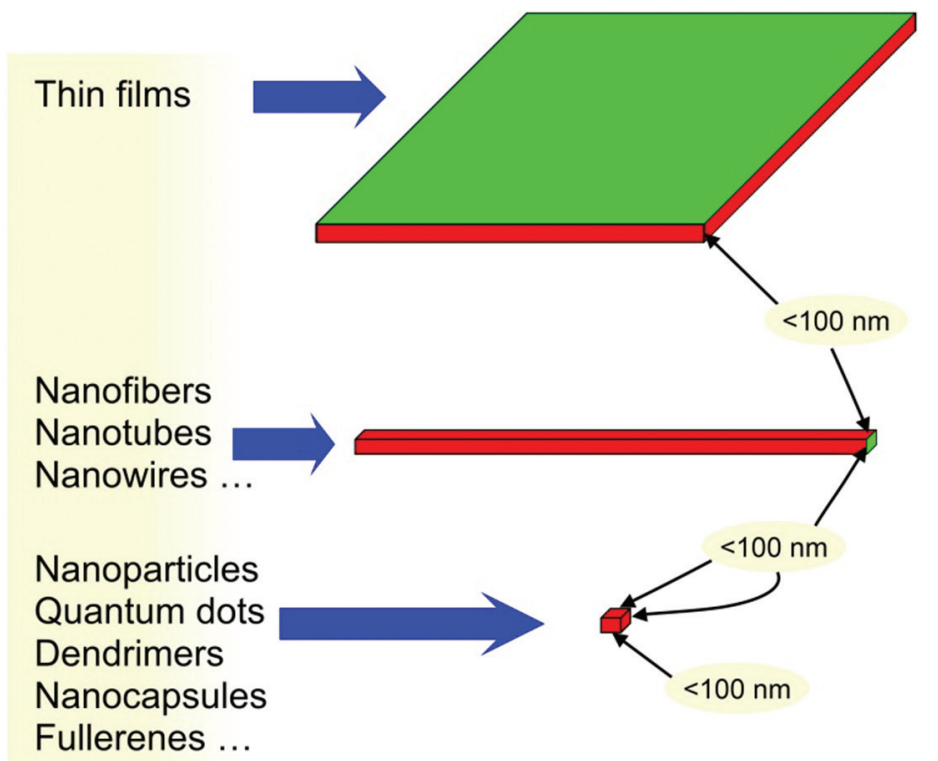

Figure 26. Some nanomaterials of interest.

In this classification, nanomaterials include:

- Materials such as thin films or surface coatings that are nanoscale in one dimension and are extended in the other two dimensions.

- Materials such as nanofibers, nanowires and nanotubes that are nanoscale in two dimensions and extended in the third dimension.

- Materials such as particles or molecules that are nanoscale in all three dimensions.

- Larger nano-crystalline materials composed of nanometer-sized pieces.

Man-made nanomaterials existed before people talked about nanotechnology. One of the examples developed over several decades, is ultra-thin films. The advantage of coating a surface with nanoparticles is that less material is needed to make the film and a better contact is realized. This is illustrated in figure 27 showing a monolayer of particles. Small particles provide a better surface coverage with less material than larger particles.

To show the importance of using ultra thin films in terms of material savings, suppose that we want to deposit a liquid film of one nanometer thickness on the surface Paris city. The question is: how much volume of liquid do we need? The area of Paris city is $105.4 \mathrm{~km}^{2} \approx 10^{26} \mathrm{~nm}^{2}$. A volume of liquid of $10^{26} \mathrm{~nm}^{3}=0.1 \mathrm{~m}^{3}=100$ liters is enough to do that. This simple 


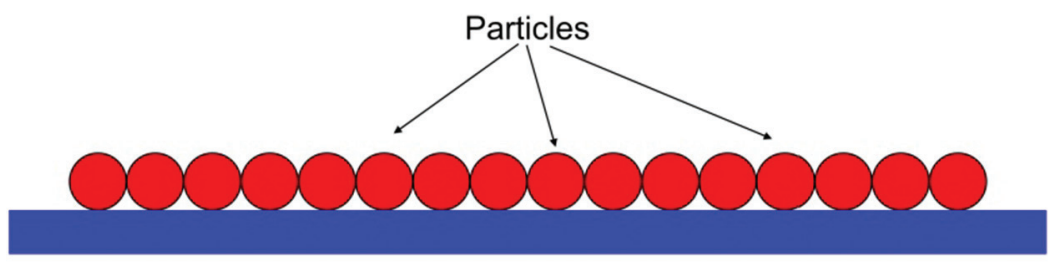

Much smaller particles

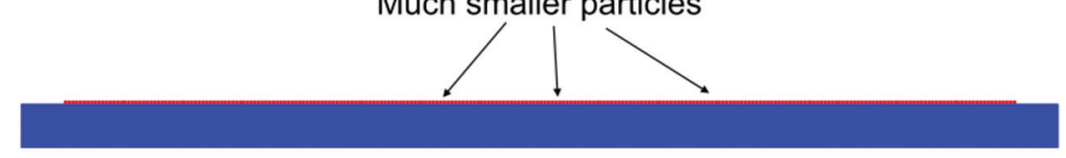

Figure 27. Difference between a monolayer made of particles (full circles) of different sizes coated on a surface.

example shows that the amount of material needed to cover Paris city is pretty small. Using nanosized films instead of microsized films, with the same properties, saves a lot of material and is a good for sustainable development.

Another way to classify nanomaterials is to focus attention on the internal structure of a macroscopic material and especially on the size of the nanostructures which are embedded within it (figure 28). In this classification, a structure has a non-zero dimension if one of its dimension at least is larger than $100 \mathrm{~nm}$. For example, a nanoparticle is a nanostructure with all three dimensions smaller than $100 \mathrm{~nm}$. Therefore, it is considered as a oD (zero dimension) object. A nanowire or a nanofiber has just one dimension larger than $100 \mathrm{~nm}$, consequently it is a $1 \mathrm{D}$ object. An ultra-thin film has two dimensions larger than $100 \mathrm{~nm}$ and is considered as a $2 \mathrm{D}$ nanomaterial. It is also the case of a stack $2 \mathrm{D}$ layers embedded in the bulk material. A ${ }_{3} \mathrm{D}$ material will be just a bulk containing microstructures instead of nanostructures.

\section{Nanostructuration}

Nanostructuring a material is interesting if improved properties are obtained. This can be done either by including specific nanostructures, or by an appropriate process generating nanostructures. Introducing nanostructures enhances the effects that are displayed in figure 29. It creates a large number of interfaces between the nanostructures and the bulk material, generating globally high surfaces in contact. These nanostructures, which are trapped in the bulk, show a size confinement into tiny parts of the bulk. 


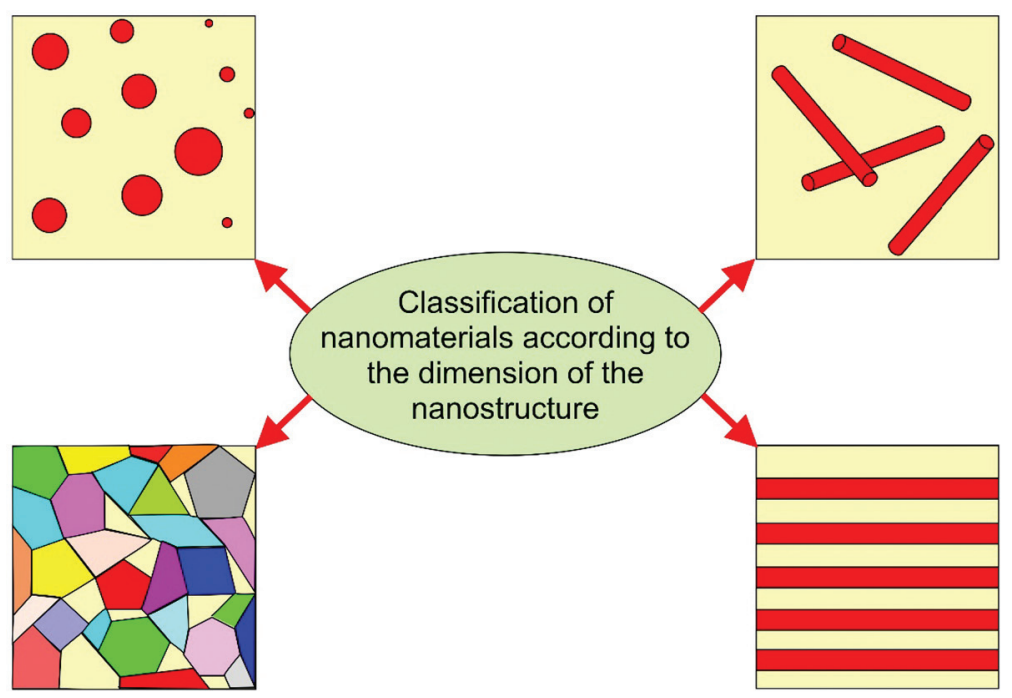

Figure 28. Classification of materials according to the dimensionality of the nanostructures. Illustration is made for $0 D, 1 D, 2 D$ and 3D nanomaterials according to this classification.

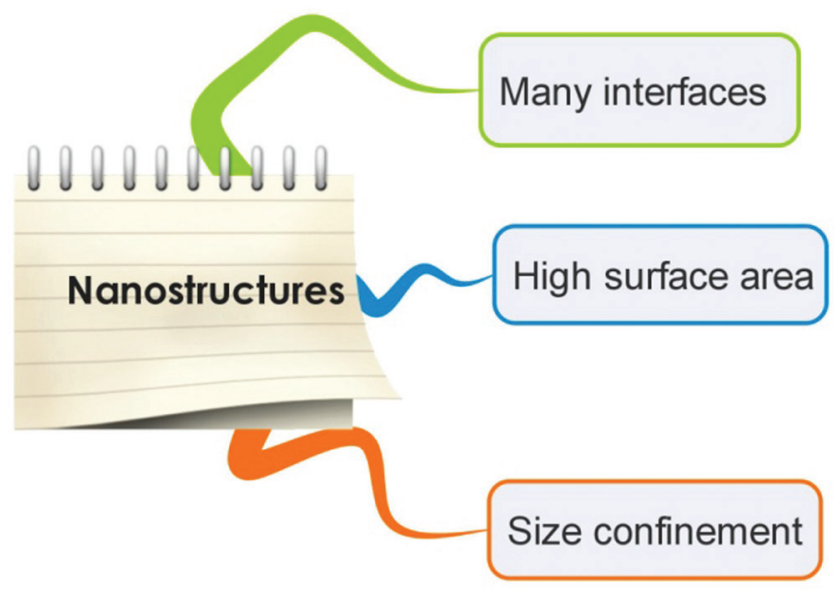

Figure 29. Properties of nanostructures bringing new features in nanostructured materials.

The size and the nature of the nanostructures which are included in a bulk material can induce major changes in the properties of the original material and improve some of the characteristics which are required for a particular application. The nanostructures can introduce specific optical or magnetic properties, for example. The nature of the nanostructure 
and the distribution of the nanostructures in the bulk material will play a key role in determining the new properties. For example, the crystallographic orientation, the chemical composition or the possibility of having out-of-equilibrium nanometer crystallites of gold or sodium chloride can lead to interesting properties of the nanostructured material. Three keypoints need to be investigated in the development of nanomaterials. They are indicated in figure 30.

Dramatic changes can be obtained. For instance, nanoparticles of black carbon included in tires improve several properties such as the ride quality and wear resistance. Adding silica smoke containing nanoparticles in concrete make it less porous and less permeable to water. This new feature is worth for concrete staying in contact with sea water.

Nanoparticles possessing specific properties can also be introduced in the bulk material. There is a great variety of characteristics which are worth to provide to the bulk material such as optical, magnetic, catalytic... properties.

Nanostructures are the building blocks of nanostructured materials. The simplest nanostructures which can be used are nanoparticles but it can be more complicated structures such as fullerene, nanocrystallites, nanotubes...

For example, by dispersing ferromagnetic grains of a size of 5 and 10 nm inside a high-resistivity amorphous film, a nanostructured film of $\mathrm{FeHf}(\mathrm{NO})$ is obtained with a high permeability and resistivity. This nanostructured material is interesting because this material can be integrated close to inductive components and minimizes parasitic capacities at high operating frequencies (up to more than $2 \mathrm{GHz}$ ).

A crystallite is a small region of solid which is a single crystal. Figure $3^{1}$ schematically shows an arrangement of several crystallites forming a polycrystalline material. Crystallites with a size in the nanometer range are called nanocrystallites. Materials used can be metals or metal oxides. For very small dimensions quantum effects can appear. Metal nanocrystalline materials are often used in heterogeneous catalysis.

An illustration of the structure of a nanocrystalline material is displayed in figure 32. Nanocrystals are embedded into an amorphous matrix.

In this chapter we shall focus our attention on some types of nanomaterials only, and postpone to next chapter the presentation of carbon-based nanostructured materials. 


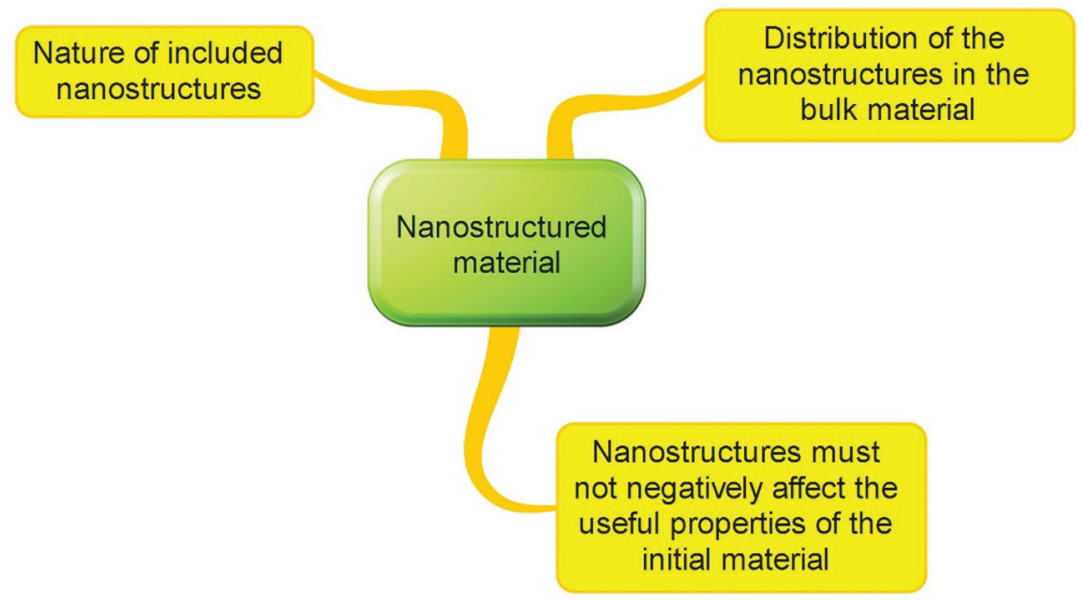

Figure 30. Three keypoints of nanostructured materials. Nanostructures included in materials can give to the nanostructured material new properties that depend on the nature of the nanostructures as well as on their geometrical distribution inside the material. Care should be taken not to negatively affect the initial properties of the material which are useful to the designed application.

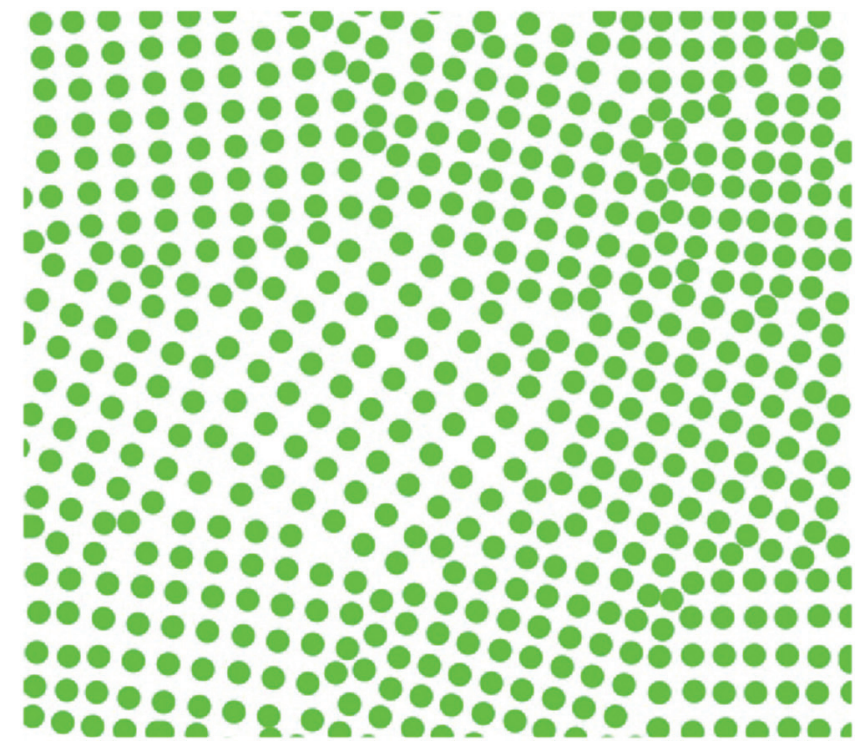

Figure 31. Schematic representation of polycrystalline material consisting of crystallites. Image from Wikimedia Commons (http://commons.wikimedia.org). Author Edward Pleshakov. 


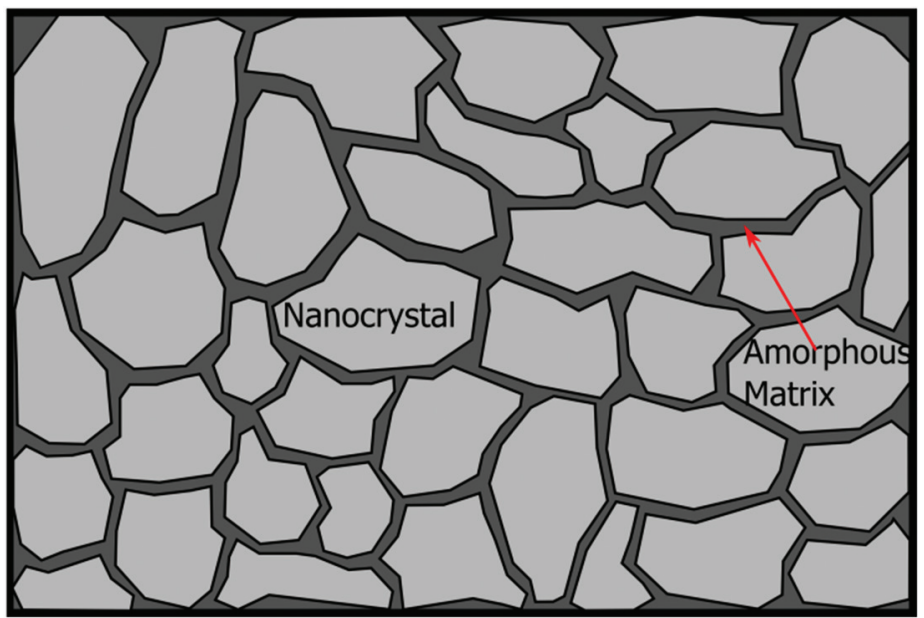

Figure 32. Schematic structure of a nanocomposite material with a nanocrystalline component embedded within an amorphous matrix. Image from Wikimedia Commons (http://commons. wikimedia.org). Author Freundchen.

\section{Dendrimers}

A dendrimer is a macromolecule made up of monomers which are assembled in a tree structure around a central core. It is a highly branched ${ }_{3} \mathrm{D}$ structure with the ability to encapsulate nanoparticles. Figure 33 shows schematically the construction of a $2 \mathrm{D}$ dendrimer by the commonly used divergent method. The dendrimer grows outward from a core molecule containing several reactive sites. The principle illustrated in figure 33 is the following. In the first step, the core molecule reacts with monomers containing one reactive group and two dormant groups. This generates a first-generation dendrimer. The dormant groups are activated and react with monomer molecules to give the second-step dendrimer. The synthesis is repeated until the proper size of the dendrimer is obtained.

The other way to synthesize dendrimers is the convergent method. The end groups are the starting point and the synthesis is inward. A comparison between the two ways of synthesizing dendrimer is shown in figure 34 .

Dendrimer chemistry was introduced in 1978 by F.Vögte and collaborators and the first family of dendrimers was synthesized in 1985 by D.A.Tomalia. Today it is possible to synthesize complex dendrimer structures and control the size, the shape, the surface and interior chemical functionalization. An example of dendrimer is shown in figure 35 . This molecule has 54 ferrocenes attached at the surface. 


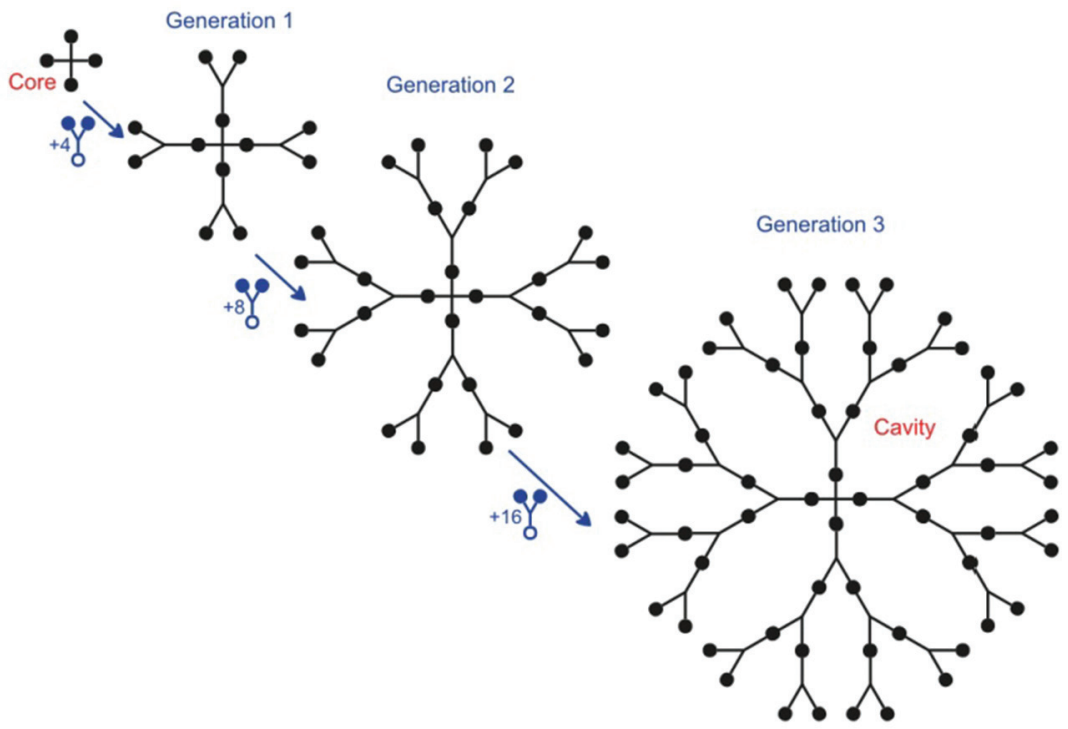

Figure 33. Schematic synthesis of a dendrimer by the divergent method.

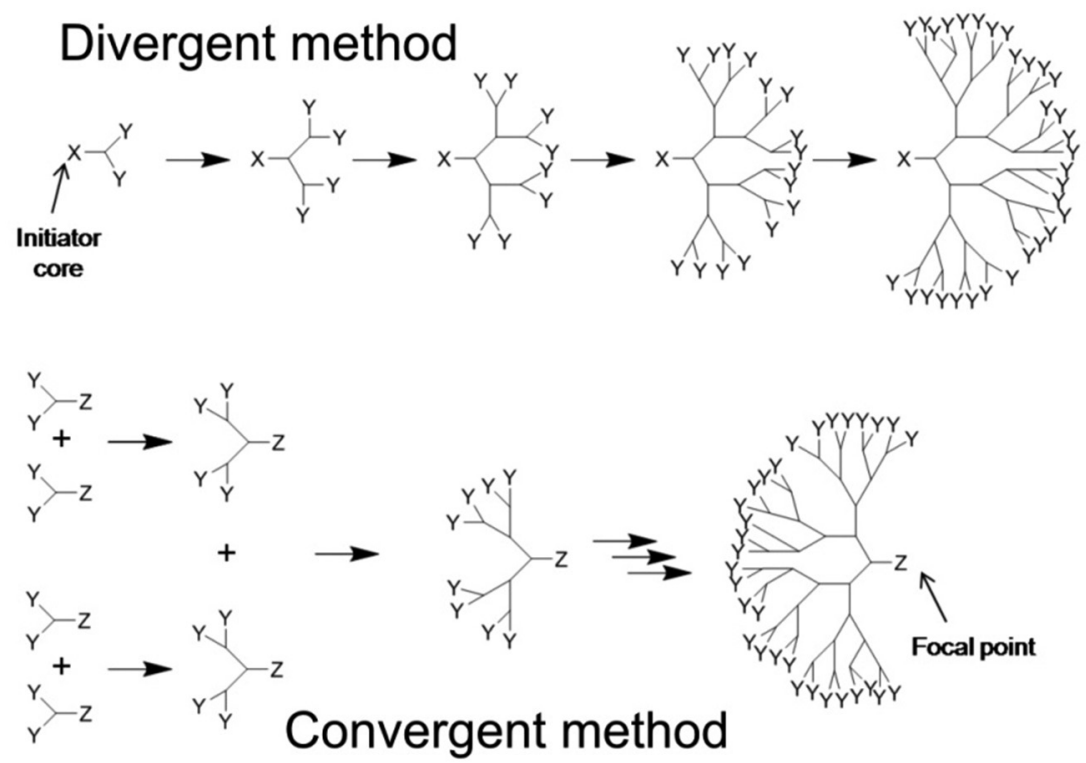

Figure 34. Principle of divergent and convergent method of synthesizing dendrimers. Image from Wikimedia Commons (http://commons.wikimedia.org). Author Chem538w10grp10. 


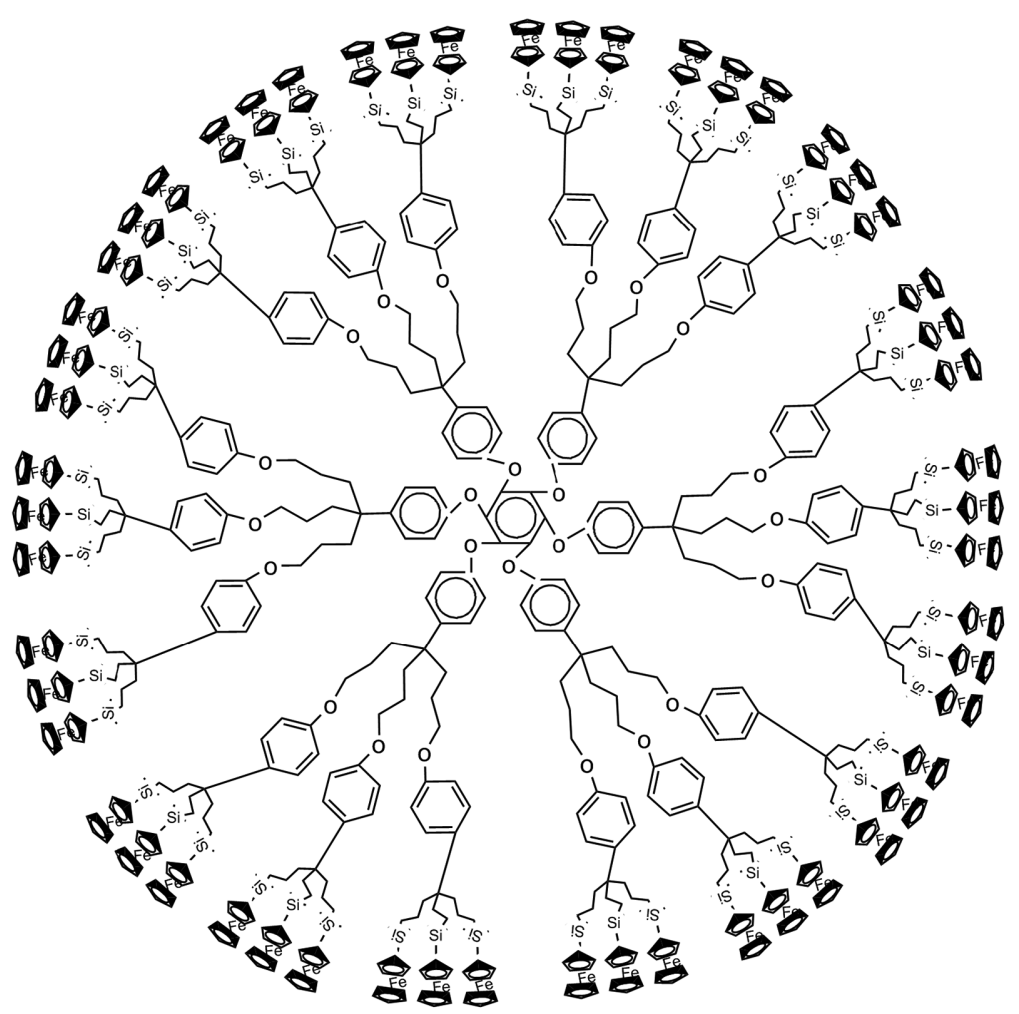

Figure 35. Dendrimer with 54 ferrocenes attached. . Image from Wikimedia Commons (http:// commons.wikimedia.org). Author Skydivermonkey.

Dendrimers can be used to carry drugs for applications such as drug delivery, diagnostics or tumor therapy. Investigations are also carried out to use dendrimers as blood substitutes.

\section{Hybrid organic-inorganic nanomaterials}

Hybrid organic-inorganic nanomaterials are interesting because they have on one side the good physical properties of ceramics and, on the other side, the convenience of organic molecules in terms of reactivity and functionalization.

Among these hybrid materials, the silsesquioxanes have interesting properties. Their stoichiometric formula reads $\mathrm{RSiO}_{3 / 2}$, where $\mathrm{R}$ is an organic radical. Their name derives from the 3/2 oxygen. Sometimes they are also called T-resins ( $\mathrm{T}$ for tri-substitued) because silicon is bonded to 3 oxygens substituants. Their most common geometrical structure is either a ladder 
or a cube. The cubic structure is commonly called the $\mathrm{T}_{8}$-cube. It has the peculiarity that the $\mathrm{O}-\mathrm{Si}-\mathrm{O}$ angle is not $90^{\circ} \mathrm{C}$ although it is often drawn like that. Figure 36 shows an example of a $\mathrm{T}_{8}$ cubic structure of silsesquioxane.

The cage structure shown in figure 36 gives the ability to put small molecules inside. Silsesquioxanes can be functionalized and used as antimicrobial coatings. Using for example quaternary ammonium salts, it is possible to make molecules which can kill bacteria and fungi while being harmless to humans and animals. Silsesquioxanes are also important for heterogeneous silica-supported transition metal catalysts.

\section{Nanocomposites}

Nanocomposite materials cover a wide domain with many applications. A nanocomposite material is a material composed of several phases where at least one of the phase has a dimension in the nanometer range. Nanoparticles included into a matrix are an example of a nanocomposite material. It is also the case of multilayers structures, which are a stack of utra-thin films of different materials. Magnetic multilayers are for example used for storage media.

Inclusion of nanoparticles in a bulk material change its physical properties. It can become stronger, lighter, more ductile... The final properties of a nanocomposite depend on many factors such as the process of manufacturing, the nature of the nanostructure and the arrangement of the nanostructure within the bulk material.

A common example of nanocomposite is a tire. In this object, carbon black nanoparticles have been added in the initial rubber. Their presence improves the strength, tensile properties, the tear and abrasion resistance... However, a too high concentration of nanoparticles in the material lowers these properties.

The concentration of nanoparticles in the initial material plays a key role in the final properties. For example, a few ppm (parts-per-million) of nanoimpureties in aluminum alloys increases substantially the strength and the corrosion resistance of aluminum.

Polymer-clay nanocomposite can improve substantially the properties of a low-cost polymer. For example they are less flammable while keeping their good mechanical properties. Such nanocomposites can be synthesized using sol-gel techniques which are realized in mild conditions. Figure 37 shows the principles of a general synthesis procedure for a nanocomposite hydrogel. Hydrogels are 3-dimensional polymer networks containing a large amount of water. They are usually fragile materials. Nanocomposite hydrogels 


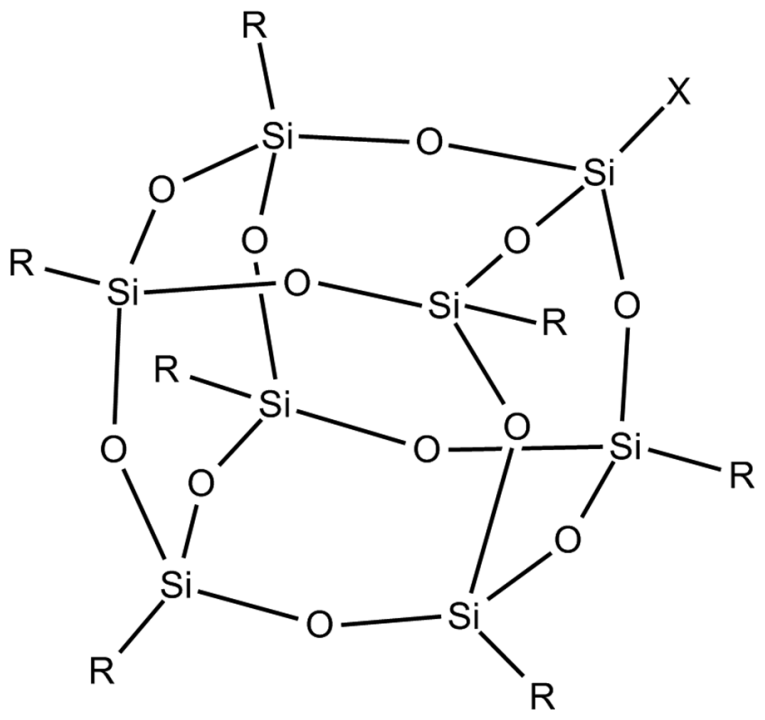

Figure 36. Example of a T8 cubic structure of Silsesquioxane. Image from Wikimedia Commons (http://commons.wikimedia.org). Author Sephirothazule.

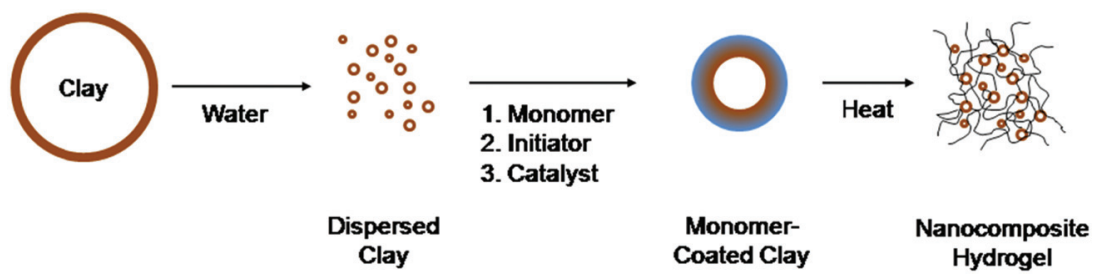

Figure 37. General synthesis procedure for a nanocomposite hydrogel. Image from Wikimedia Commons (http://commons.wikimedia.org). Author Chem538w10grp6.

synthesized with mild chemistry has made a breaktrough in this field. It is now possible to manufacture nanocomposite hydrogels with various shapes and surfaces, with high toughness, with excellent optical properties and with the ability to respond to external stimuli.

\section{Smart materials}

Smart materials are materials which can appropriately respond to stimuli provided by their environment. These materials are usually composite materials containing perception, response and control capabilities of various kinds (figure 38). External stimuli can be of various kinds: light, heat, electricity, radio waves, strain, etc. The materials are able to detect and 


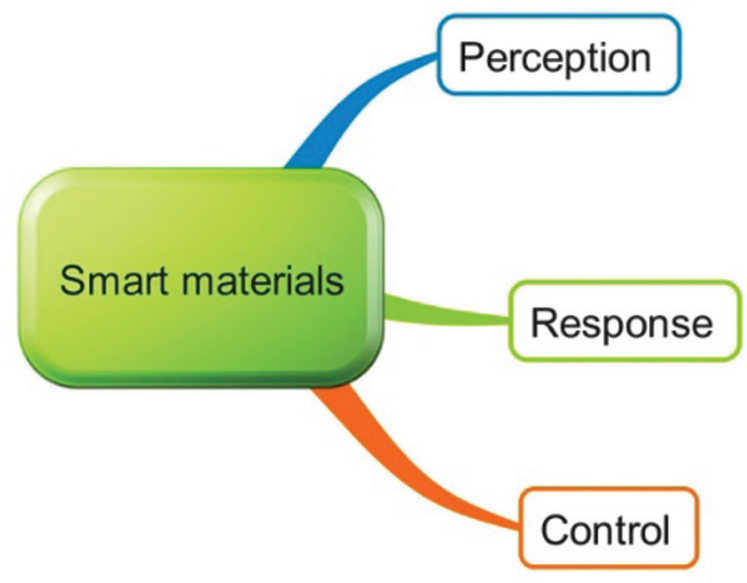

Figure 38. Main properties that a smart material should possess.

identify these stimuli above certain threshold levels and respond to them by choosing among a number of solutions decided by the designer. They should provide these responses within an appropriate time span and at the right level. The process should be reversible. After fulfilling its function, the smart material can be returned to its initial state. Some examples of smart materials are thermoelectric materials capable of converting temperature differences into electricital signals, shape-memory alloys or polymers which can return to their original shapes, and piezoelectric materials sensitive to external pressure changes. Nanotechnology provides new opportunities to fabricate smart materials.

\section{Summary}

Nanomaterials will play increasingly important roles in the future, offering new or improved properties compared to materials which are not nanostructured. Nanotechnology will be applied to build new materials incorporating a number of desired capabilities. Nanotechnology also offers the opportunity to employ less material to accomplish the same goal. This is particularly important for sustainable development because the world may soon be short of some essential raw materials if we continue to use them as we do now. A full exploitation of the potential of nanomaterials demands a dependable understanding of their properties and mechanisms on all dimensional scales. The development of multiscale models, able to predict the properties of a material on all size scales is an important goal in this field. 